ISSN 0103-9954

\title{
ESTRUTURA E COMPOSIÇÃO FLORÍSTICA DE DOIS FRAGMENTOS DA FLORESTA ESTACIONAL DECIDUAL DO ALTO-URUGUAI, SC
}

\section{FLORISTIC COMPOSITION AND STRUCTURE OF TWO SEASONAL DECIDUOUS FOREST PATCHES IN ALTO-URUGUAI, SC}

\author{
Ademir R. Ruschel ${ }^{1} \quad$ Miguel P. Guerra ${ }^{2}$ Rubens O. Nodari ${ }^{3}$

\section{RESUMO}

Em dois fragmentos florestais da Floresta Estacional Decidual (FED) do Alto-Uruguai, SC, foram aplicados os métodos de parcelas (MP) e de pontos-quadrantes (MQ) visando a comparar os métodos amostrais e caracterizar a riqueza florística e composição estrutural. Foram inventariadas 91 espécies, sendo que 52 foram encontradas no sub-bosque (sem valor madeireiro), com destaque para Sorocea bonplandii, Gymnanthes concolor e Trichilia claussenii. As outras 39 espécies emergentes no dossel florestal são de uso madeireiro, sendo as mais abundantes Apuleia leiocarpa, Nectandra megapotamica, Cabralea canjerana e Holocalyx balansae. As espécies de maior densidade e área basal foram praticamente as mesmas em ambos os fragmentos florestais, independente do método usado. O MP detectou uma maior diversidade de espécies e uma maior densidade de plantas por área comparativamente ao $\mathrm{MQ}$, embora ambos os métodos detectassem espécies diferentes. Todavia, o uso simultâneo dos dois métodos possibilitou em uma maior cobertura da diversidade de espécies nas duas áreas, com redução no tempo para o inventário. Em ambos os métodos, detectaram-se erros amostrais acima do esperado e quanto menores os valores do erro amostral, maior foi a similaridade dos resultados das avaliações entre os métodos. Paradoxalmente a riqueza de espécies presentes na FED contrasta com o pequeno número de fragmentos que restaram neste ecossistema. Importante ressaltar que esses fragmentos são as últimas fontes de sementes para a recolonização de áreas abandonadas ou degradadas, como também para conservação e uso em programas de melhoramento genético de essências florestais nativas e banco de sementes in situ.

Palavras-chave: espécies madeireiras; floresta subtropical atlântica; inventário florestal; riqueza de espécies.

\section{ABSTRACT}

In two forest remnants located in Seasonal Deciduous Atlantic Forest of Alto-Uruguai region of Southern Brazil, the plots (MP) and point-centered-quarter (MQ) methods were used aiming to characterize the floristic richness and structural composition. 91 species were identified, 52 of them were classified as understory species (without timber value), being the most abundant Sorocea bonplandii, Gimnanthes concolor and Trichilia claussenii species. The others were classified as timber species, being the most abundant Apuleia leiocarpa, Nectandra megapotamica, Cabralea canjerana, and Holocalyx balansae. The species of higher density and basal area were practically the same in both forest patches, independently from the inventory method used. The MP detected a higher number of species diversity as well as the higher value for plant density by area, comparatively to MQ, although both methods detected different species. The simultaneous use of MQ and MP resulted in a higher coverage of species diversity in both areas, with a reduction in time to perform the inventory. In both methods, sampling error above of the expected value was detected, but the smaller these values, the greater the similarity among the results provided by both methods. Unfortunately, the species richness occurring in the FED contrasts with the small number of remaining patches in this ecosystem. It is important to highlight that these patches are the last repositories for seed to be used in colonizing degraded areas as well as for conservation, improvement and in situ seed bank program with native plant species.

Keywords: floristic richness; forest inventory; subtropical Atlantic forest; timber species.

\section{INTRODUÇÃO}

No sul do Brasil, no âmbito do bioma Mata Atlântica são reconhecidas três tipologias florestais: a

1. Engenheiro Agrônomo, Dr., Pesquisador Embrapa Amazônia Oriental, Trav. Dr. Enéas Pinheiro s/nº C.P. 48, CEP 66095-100, Belém-PA, Brasil,. E-mail: ruschel@cpatu.embrapa.br

2. Engenheiro Agrônomo, Dr., Professor Titular do Programa de Pós-Graduação em Recursos Genéticos Vegetais. UFSC, Caixa Postal 476, CEP. 88040-900. Florianópolis, SC. E-mail: mpguerra@cca.ufsc.br

3. Engenheiro Agrônomo, Dr., Professor Titular do Programa de Pós-Graduação em Recursos Genéticos Vegetais. UFSC, Caixa Postal 476, CEP. 88040-900. Florianópolis, SC. E-mail: nodari@cca.ufsc.br

Recebido para publicação em 8/03/2007 e aceito em 6/05/2009. 
Floresta Ombrófila Densa, estendendo-se ao longo da região litorânea em altitudes inferiores a 500 m; a Floresta Ombrófila Mista, caracterizada pela predominância do pinheiro-do-paraná e ocorrendo em altitudes geralmente superiores a $500 \mathrm{~m}$, na região do planalto e por final, a Floresta Estacional Decidual (FED), que compreende as florestas das porções médias e superiores do vale do Uruguai ocorrendo em altitudes superiores a $200 \mathrm{~m}$ e inferiores a $600 \mathrm{~m}$ (REITZ et al., 1978; IBGE, 1990). A FED, ainda é conhecida como Floresta Subtropical Atlântica, ou Floresta Pluvial do Alto-Uruguai (RAMBO, 1956; KLEIN, 1972).

A FED é a tipologia florestal mais ameaçada e menos protegida da Mata Atlântica no sul do Brasil, encontrando-se completamente fragmentada e restando menos de 4,2\% de sua área original (IBGE, 1990). Esses fragmentos representam os últimos refúgios da exuberante riqueza de espécies da flora e da fauna da Floresta Subtropical Atlântica (WALLAUER E ALBUQUERQUE, 1986; RUSCHEL et al., 2005), sendo também a tipologia florestal menos conhecida e estudada.

A ocupação da Floresta Estacional Decidual na região oeste catarinense ocorreu a partir de 1917, por frentes pioneiras de colonização de imigrantes provenientes do Rio Grande do Sul (italianos, alemães), estendendo-se até os anos 50 (PIAZZA, 1994). A exploração dos recursos naturais existentes promoveu um aumento expressivo das exportações catarinenses em seis vezes. No decorrer dos anos, a produção de madeira aumentou 22 vezes e a da erva-mate cerca 13 vezes, sendo que a maioria das exportações (madeira, erva-mate) foi escoada via Rio Uruguai para a Argentina e Uruguai (PIAZZA, 1994). Além disso, o autor ainda menciona que houve aumento dos produtos agrícolas como um todo (feijão, banha, manteiga, arroz, queijo).

A FED apresenta uma alta diversidade de espécies lenhosas, sendo que Reis (1993) reportou 181 espécies e Ruschel et al. (2005) em um levantamento em 13 fragmentos florestais inventariaram 57 espécies de uso madeireiro, as quais totalizaram $64 \%$ das espécies lenhosas e $36 \%$ da densidade florestal. No entanto, caracterizar a composição florística e estrutural dos remanescentes da FED é importante para o manejo e conservação destes. Todavia, os remanescentes da FED do Alto-Uruguai encontram-se ameaçados, por causa da construção de hidrelétricas, as quais vêm substituindo esses poucos e ímpares remanescentes florestais por lagos das represas. A principal estratégia para amenizar as perdas dessa biodiversidade tem sido a criação de reservas florestais próximos a esses empreendimentos hidrelétricos (áreas de compensação ambiental), o que é legalmente exigido. Nesse contexto, conhecer a composição estrutural e a riqueza dessas espécies, dos poucos remanescentes é importante para subsidiar esta estratégia e direcionamentos nos programas de compensação ambiental, bem como de outras estratégias de manejo e conservação desta tipologia florestal. Embora se necessite também conciliar o conhecimento da ecologia dessas espécies, para assim permitir que a regeneração natural e ou induzida avance em todos os estádios sucessionais.

No presente estudo, buscou-se caracterizar a composição florística e estrutural de dois fragmentos florestais no Alto Uruguai em Santa Catarina, bem como verificar a eficácia dos métodos de amostragem de parcelas e pontos-quadrantes, utilizados para obter maior cobertura ao inventário.

\section{MATERIAL E MÉTODOS}

\section{Localização da área}

Os inventários florísticos foram realizados em dois fragmentos da Floresta Estacional Decidual - FED do Alto-Uruguai, localizados nos municípios de Itapiranga, SC, distrito de Sede Capela Colégio (CCO), em altitude de $320 \mathrm{~m}$, latitude $27^{\circ} 11^{\prime} 25^{\prime \prime} \mathrm{S}$ e longitude $53^{\circ} 38^{\prime} 02^{\prime \prime} \mathrm{W}$ e em Descanso, SC, distrito de São Valentin (SVA), em altitude de $590 \mathrm{~m}$, latitude $26^{\circ} 56^{\prime} 12^{\prime \prime} \mathrm{S}$ e longitude $53^{\circ} 31^{\prime} 30^{\prime \prime} \mathrm{W}$. Conforme Uberti (2005), a região edafo-ambiental é homogênea, sendo que a maioria absoluta dos solos têm cor vermelha e textura argilosa, os solos são eutróficos e originados de basalto amigdaloidal e bem drenados. Sua taxonomia restringe-se a Nitossolos, Cambissolos, Chernossolos e Neossolos Litólicos. O relevo dominante é o fortemente ondulado e montanhoso.

O clima é do tipo Cfa (Köppen), sem período seco definido e com alta intensidade e regularidade pluviométrica. A precipitação média anual é de $1.878 \mathrm{~mm}$. Os índices térmicos da região determinam dois períodos bem distintos: um período de quatro a cinco meses com médias compensadas iguais ou superiores a $20^{\circ} \mathrm{C}$ e outro com duração de dois a três meses com médias iguais ou inferiores de $15^{\circ} \mathrm{C}$ (Julho $13,5^{\circ} \mathrm{C}$ ). $\mathrm{O}$ clima, apesar de quente-úmido durante boa parte do ano, conserva, por apreciável período, caráter frio, capaz de imprimir restrições à proliferação e o desenvolvimento de um grande número de espécies tipicamente tropicais 
(IBGE, 1990).

Os inventários florestais foram realizados em dois fragmentos florestais, ambos com área aproximadamente de 50 ha pelos métodos de pontos quadrantes - MQ (COTTAM e CURTIS, 1956) e de parcelas - MP (MÜLLER-DOMBOIS e ELLEMBERG, 1974). A amostragem dentro dos fragmentos florestais foi alocada sobre a diagonal maior do remanescente e a partir dos $20 \mathrm{~m}$ medidos da borda da floresta estabeleceu-se o limite periférico para o inventário. O número de unidades amostrais no MP foi preestabelecido em sete parcelas e para MQ este número foi limitado pela quantidade possível de unidades amostrais (pontos) sobre a maior diagonal do remanescente. Os pontos amostrais foram distanciados de $15 \mathrm{~m}$, estabelecidos alternadamente a direita e a esquerda da diagonal e afastados perpendicularmente $5 \mathrm{~m}$ desta. No MP, foram instaladas sete parcelas de forma sistematizada em cada um dos fragmentos, distribuídas eqüidistantes $30 \mathrm{~m} \mathrm{e}$ alternadas em relação à posição da linha de maior diagonal do fragmento, cada parcela resultando em área de $1.600 \mathrm{~m}^{2}$ (40 m x $\left.40 \mathrm{~m}\right)$. Em ambos os métodos, toda planta que apresentou DAP $\geq 5 \mathrm{~cm}$ foi incluída na amostragem. No MQ, adicionalmente, no mesmo ponto, avaliou-se em cada quadrante a planta de uso madeireiro, o que permitiu uma segunda análise da estrutura das espécies com uso madeireiro.

\section{Coleta dos dados}

Cada planta amostrada foi identificada taxonomicamente, etiquetada, sendo mensurado o DAP, a distância da planta ao ponto central, altura do fuste (AF) e a altura total (AT). A AF foi mensurada a partir da base do solo até a primeira bifurcação e AT a partir da base do solo ao final da copa, com o auxílio de régua dendrométrica ou clinômetro. A identificação taxonômica foi efetuada em campo quando inequívoca, adotandose o sistema de classificação de Cronquist (1988). Em casos de dúvida, foi feita a coleta de exsicatas compostas de partes reprodutivas e vegetativas, sendo a identificação feita com auxílio de bibliografia especializada (REITZ et al., 1978; LORENZI, 1998) e com apoio dos botânicos Dr. Ademir Reis (USFC) e Dr. Marcos Sobral (UFMG). Após a identificação, as espécies foram classificadas nas categorias espécies de valor madeireiro no comércio e espécies sem valor madeireiro (RUSCHEL et al., 2003).

\section{Análise dos dados}

No MQ, a densidade de plantas (plantas $\mathrm{ha}^{-1}$ ) foi estimada com base na média da área das distâncias individuais das plantas amostradas ao ponto (KREBS, 1989). O erro amostral foi estimado com base ao erro padrão das distâncias individuais das plantas amostradas (POLLARD, 1971). No método de parcelas, a densidade foi estimada pela média de plantas obtida nas parcelas e para a estimativa do erro amostral usou-se o modelo proposto por Krebs (1989) e adicionalmente a suficiência de amostragem foi determinada pela Curva do Coletor (BRAUN-BLANQUET, 1979).

Partindo dos parâmetros dendrométricos e fitossociológicos (DAP, AT, AF, número de plantas, espécies, famílias), foram realizadas as análises descritivas tais como: médias aritmétricas, percentagens, área basal, índices da diversidade Shannon-Wienner $\left(\mathrm{H}^{\prime}\right)$, recíproca de Simpson's ( $\left.\mathrm{Sr}^{\prime}\right)$, equabilidade comunitária $\left(\mathrm{J}^{\prime}\right)$ e os índices de similaridade Jaccard (IsJ), Morisita (IsM'), Bray-Curtis (IsBC') e Percentagem simples (IsSp') de acordo com Magurran (1988) e Krebs (1989).

\section{RESULTADOS E DISCUSSÃO}

Para ambos os métodos, observou-se o ingresso crescente de novas espécies com o aumento de unidades amostrais, sugerindo que a riqueza de espécies nos fragmentos CCO e SVA ainda é superior à revelada pelos inventários. Da mesma maneira, foi detectada uma maior riqueza de espécies no fragmento SVA, comparada ao fragmento CCO (Figura 1). 

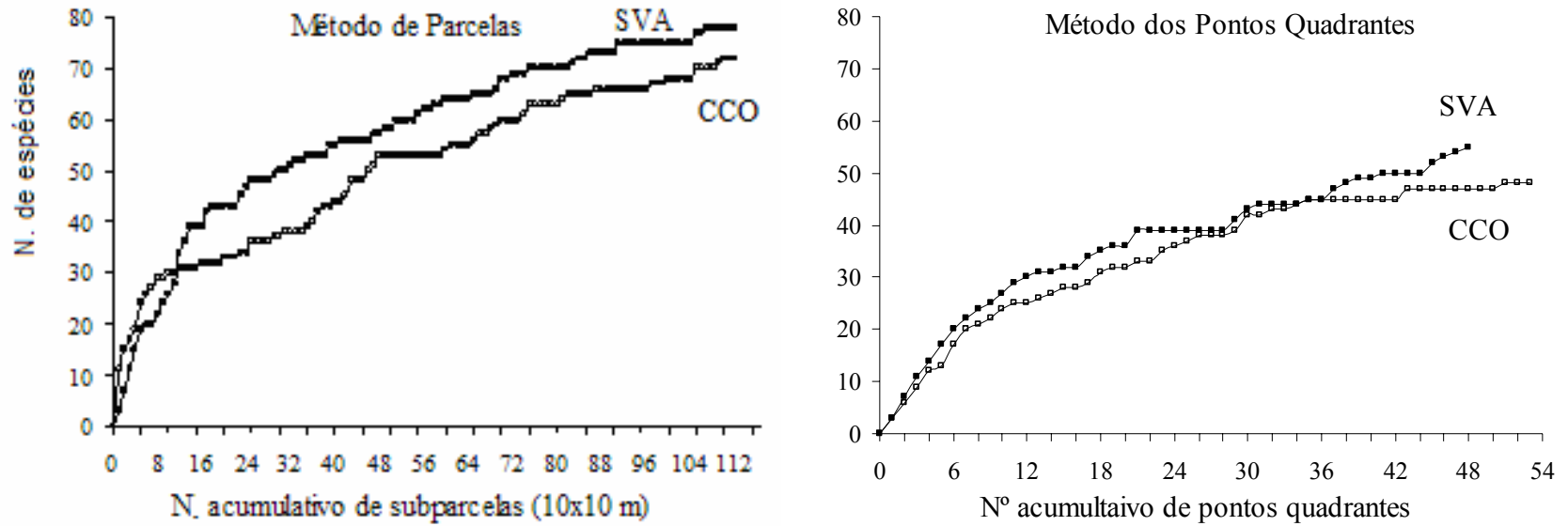

FIGURA 1: Número cumulativo de espécies por unidade amostral partindo de plantas arbóreas com DAP $\geq 5$ $\mathrm{cm}$, amostradas pelos métodos de parcelas e pontos-quadrantes, nos fragmentos São Valentin (SVA) e Capela Colégio (CCO) da Floresta Estacional Decidual do Alto-Uruguai, SC.

FIGURE 1: Cumulative species-area curves for the woody plants with a $\mathrm{DBH} \geq 5 \mathrm{~cm}$ sampled with the use of quadrate plots and the point centered quarter methods in the São Valentin (SVA) and Capela Colégio (CCO) fragments of the Seasonal Deciduous Forest, Alto-Uruguai, SC.

A insuficiência amostral também foi observada nas estimativas do erro amostral, os quais variaram de $6,6 \%$ a $33 \%$. Em CCO o método de parcelas revelou que o maior erro amostral esteve associado à variação no grupo das espécies de uso madeireiro, enquanto que em SVA os dois métodos detectaram grandes variações nos valores dos erros amostrais, mesmo para o subgrupo de espécies madeireiras (Tabela 1). Assim, parte das diferenças nos resultados obtidos dentro do mesmo fragmento pode ser atribuída ao erro amostral. Isto porque houve maior similaridade nos resultados quando o erro amostral foi baixo para ambos os métodos, o que foi constatado no fragmento $\mathrm{CCO}$, onde a densidade de plantas estimadas por ambos os métodos foi muito próximo, 1116 (MP) e 1012 (MQ). Contrariamente quanto maior o erro amostral mais discrepantes foram os resultados. Assim, a estimativa do erro amostral é de alta relevância em inventários florestais e deve ser um dos critérios que elevam a precisão das avaliações, independentemente do método empregado.

TABELA 1: Parâmetros dendrométricos das plantas com DAP $\geq 5 \mathrm{~cm}$ nas categorias todas as espécies (Todas) e espécies madeireiras (Mad) obtidos pelos métodos pontos quadrantes (MQ) e parcelas (MP) nos fragmentos São Valentin (SVA) e Capela Colégio (CCO) da Floresta Estacional Decidual do Alto-Uruguai, SC.

TABLE 1: Dentrometry parameters of the woody plants with $\mathrm{DBH} \geq 5 \mathrm{~cm}$ in the groups of all species (Todas) and timber species (Mad) evaluated through the quadrate plots (MP) and point-centered quarter methods (MQ), in the São Valentin (SVA) and Capela Colégio (CCO) fragments of the Seasonal Deciduous Forest in Alto-Uruguai, SC.

\begin{tabular}{|c|c|c|c|c|c|c|c|c|}
\hline \multirow{3}{*}{ Parâmetros dendrométricos } & \multicolumn{4}{|c|}{ Fragmento $\mathrm{CCO}$} & \multicolumn{4}{|c|}{ Fragmento SVA } \\
\hline & \multicolumn{2}{|c|}{ MP } & \multicolumn{2}{|c|}{$\mathrm{MQ}$} & \multicolumn{2}{|c|}{ MP } & \multicolumn{2}{|c|}{$\mathrm{MQ}$} \\
\hline & Todas & Mad & Todas & Mad & Todas & Mad & Todas & Mad \\
\hline N. unidades amostrais & 7 & 7 & 52 & 44 & 7 & 7 & 48 & 38 \\
\hline N. plantas amostradas & 1296 & 286 & 208 & 176 & 1119 & 441 & 192 & 152 \\
\hline Erro amostral (\%) & 12,2 & 27,7 & 12,5 & 28,6 & 33,0 & 6,6 & 17,1 & 30,0 \\
\hline N. de espécies & 69 & 29 & 43 & 18 & 75 & 32 & 51 & 28 \\
\hline N. de plantas $\left(\right.$ ha $\left.^{-1}\right)$ & 1116 & 234 & 1012 & 212 & 982 & 340 & 677 & 285 \\
\hline Área basal $\left(\mathrm{m}^{2} \mathrm{ha}^{-1}\right)$ & 33,9 & 23,6 & 41,5 & 32,5 & 32,8 & 26,4 & 32,9 & 28,5 \\
\hline DAP médio $(\mathrm{cm})$ & 13,3 & 25,7 & 15,9 & 25,6 & 14,6 & 24 & 18,4 & 24,1 \\
\hline Altura total média (m) & 8,5 & 12,7 & 9,5 & 11,9 & 9,1 & 12,4 & 10,5 & 12,7 \\
\hline Altura comercial média (m) & & 5,7 & & 6,5 & & 6,9 & & 7,2 \\
\hline
\end{tabular}


No grupo das espécies madeireiras e de subosque (não madeireiras), detectaram-se diferenças consideráveis entre os dois fragmentos, enquanto que em $\mathrm{CCO}$, ambos os métodos proporcionaram valores similares para as duas categorias. Em CCO as espécies madeireiras representaram nos dois métodos um quinto (21\% no MP e 20,9\% MQ) da densidade de plantas (Tabela 1). Porém, em SVA as estimativas da densidade e mesmo do número de espécies madeireiras variaram fortemente entre os métodos. Assim, a densidade de plantas de uso madeireiro em SVA foi dois terços superiores a CCO (34,6\% no MP e 42,1\% no $\mathrm{MQ}$ ), porém menos discrepante para o número de espécies (40,6\% no MP e 42,7\% MQ). As espécies madeireiras representaram a maior parte da área basal da floresta, pois em $\mathrm{CCO}$, os valores alcançaram mais de dois terços do total $(69,6 \%$ no MP e $78,3 \%$ MQ) e, em SVA, mais de quatro quintos $(80,5 \%$ no MP e $86,6 \%$ no MQ).

Os dois fragmentos avaliados apresentaram altos valores para riqueza de espécies, pois foram detectadas 42 famílias e 91 espécies nesses dois fragmentos. O método MP se mostrou mais eficiente na análise da riqueza de espécies (Tabela 1). Em CCO foram identificadas 79 espécies, sendo 69 espécies detectadas pelo MP e 43 espécies pelo MQ. Considerando que quarenta espécies foram detectadas conjuntamente, o MP foi o mais eficiente dos métodos utilizados, ao detectar, além das quarenta espécies em comum, 29 outras comparativamente a três espécies detectadas pelo MQ. Já, no fragmento SVA, foram identificadas 79 espécies, sendo 75 detectadas pelo MP e 51 pelo MQ. Destas, 47 espécies foram comuns aos dois métodos. Novamente o MP foi mais eficiente, pois detectou 28 espécies adicionais as 47 comuns, enquanto o MQ detectou somente quatro (Tabela 2). A análise também revelou que da diversidade florística de 91 espécies detectadas nos dois fragmentos, 31 espécies não foram compartilhadas, sendo, 19 espécies exclusivas em SVA e 12 em CCO.

Os valores médios das características diâmetro, altura total, comprimento do fuste e área basal apresentaram diferenças entre as metodologias. Os valores oriundos do MQ foram superiores àqueles demonstrados pelo MP. Novamente a maior similaridade entre métodos e também entre fragmentos foi observada para a categoria das plantas madeireiras (Tabela 1). Os resultados foram muito similares entre os dois métodos nas análises mais generalizadas, como para espécies dominantes no fragmento florestal ou, densidade de plantas e ou proporcionalidades de plantas e espécies por categoria de espécies. Tais constatações estão em conformidade com os índices de diversidade e similaridade. O método de pontos quadrantes (MQ) é considerado prático e informativo para inventários florísticos, sendo vantajoso em termos de tempo e custos, embora não seja recomendável para o estudo individualizado por espécie (POLLARD, 1971; GIBBS et al., 1980; KREBS, 1989; DIAS et al., 1992; MARTINS, 1993; METZGER et al., 1998; SILVA et al., 2002; SPARKS et al., 2002). Sparks et al. (2002) compararam seis diferentes metodologias para inventários florísticos e observaram maior ajuste proporcionado pelos métodos de círculos com raios fixos e de parcelas quadradas.

Florísticamente, em ambos os métodos, as espécies Sorocea bonplandii, Calyptranthes tricona, Trichilia claussenii e Gymnanthes concolor exibiram maior densidade de plantas no CCO. No fragmento SVA observou-se padrão similar com exceção de Calyptranthes tricona e Chrysophyllum gonocarpum (Tabela 2). Estas espécies são ciófitas e características do subosque florestal e servem de padrão para o reconhecimento da tipologia florestal, mesmo porque a presença destas espécies caracteriza o estádio sucessional avançado da formação florestal (RUSCHEL et al., 2006).

Entre as espécies identificadas, várias se encontram na lista das espécies ameaçadas de extinção no Rio Grande do Sul (CONSEMA, 2002), onde a tipologia Floresta Estacional Decidual (FED) cobre grande porção do Estado, constam: Apuleia leiocarpa, Aralia warmingiana, Jacaranda puberula, Jacaratia spinosa, Myrocarpus frondosus e Picrasma crenata. Além disso, várias espécies apresentaram baixa densidade florestal, dentre elas: Hennecartia omphalandra, Strychnos brasiliensis, Achatocarpus praecox, Pilocarpus pennatifolius, Helietta apiculata, Styrax leprosus, Myrcianthes pungens, Maclura tinctoria, Ruprechtia laxiflora e Eugenia rostrifolia. Esses resultados indicam a necessidade de especial atenção e a tomada de medidas eficazes para a conservação dessas espécies de baixa densidade, muitas praticamente desconhecidas e ou que ainda não receberam a devida importância, sendo assim, as espécies mais suscetíveis a erosão genética e extinção local. Embora a flora arbórea da FED seja representada por 3,6\% de espécies exclusivas, $31,4 \%$ preferenciais e $65,0 \%$ de baixa representatividade, comuns a outros ecossistemas (IBGE, 1990), estão distribuídas em poucos fragmentos, o que demanda esforços consideráveis para sua conservação. 
TABELA 2: Densidade absoluta de plantas $\left(\mathrm{ha}^{-1}\right)$ com DAP $\geq 5 \mathrm{~cm}$ agrupadas por famílias, espécies e classe de uso (M- com valor madeireiro; S- sem valor madeireiro) avaliadas pelos métodos pontos-quadrantes (MQ) e parcelas (MP) nos fragmentos São Valentin (SVA) e Capela Colégio (CCO) da Floresta Estacional Decidual do Alto-Uruguai, SC.

TABLE 2: Absolute density of plants $\left(\mathrm{ha}^{-1}\right)$ with $\mathrm{DBH} \geq 5 \mathrm{~cm}$ grouped by families, species and class of use (M- with timber value; S- without timber value), using sampling point-quarter centered (MQ) and quadrate plots methods (MP) in the São Valentin (SVA) and Capela Colégio (CCO) fragments of the Seasonal Deciduous Forest in Alto-Uruguai, SC.

\begin{tabular}{|c|c|c|c|c|c|c|}
\hline \multirow{2}{*}{ Família } & \multirow{2}{*}{ Nome científico } & \multirow{2}{*}{$\begin{array}{l}\text { Classe de } \\
\text { uso }\end{array}$} & \multicolumn{2}{|c|}{$\mathrm{CCO}$} & \multicolumn{2}{|c|}{ SVA } \\
\hline & & & MQ & MP & MP & MQ \\
\hline Achatocarpaceae & Achatocarpus praecox Griseb. & $\mathrm{S}$ & - & 2,2 & - & - \\
\hline \multirow{2}{*}{ Anonaceae } & Rollinia cf. silvatica (A. St.-Hil) Mart. & S & - & 2,2 & 4,9 & - \\
\hline & Rollinia rugulosa Schltdl. & S & - & - & 1,0 & - \\
\hline \multirow{2}{*}{ Apocynaceae } & Aspidosperma parvifolium A. DC. & M & - & - & 4,9 & 3,4 \\
\hline & Rauvolfia sellowii Müll. Arg. & $\mathrm{S}$ & - & 2,2 & 2,0 & 3,4 \\
\hline \multirow[b]{2}{*}{ Araliaceae } & Aralia warmingiana (Marchal) J. Wen & $\mathrm{M}$ & - & 2,2 & 4,9 & 3,4 \\
\hline & $\begin{array}{l}\text { Schefflera morototoni (Aubl.) Maguire, } \\
\text { Steyerm. \& Frondin }\end{array}$ & M & - & 2,2 & 1,0 & - \\
\hline Arecaceae & Syagrus romanzoffiana (Cham.) Glassm. & $\mathrm{S}$ & - & 4,5 & 16,7 & 18,4 \\
\hline Bignoniaceae & Jacaranda puberula Cham. & $\mathrm{M}$ & - & - & 2,9 & 3,4 \\
\hline Bombacaceae & Chorisia speciosa A. St.-Hil. & $\mathrm{S}$ & - & 2,2 & 4,9 & 3,4 \\
\hline \multirow{3}{*}{ Boraginaceae } & Cordia ecalyculata Vell. & $\mathrm{M}$ & - & 2,2 & 1,0 & - \\
\hline & Cordia trichotoma (Vell.) Arráb. ex Steud. & M & 5,1 & 4,5 & 4,9 & 3,4 \\
\hline & Patagonula americana L. & M & - & 2,2 & 1,0 & 3,4 \\
\hline Caricaceae & Jacaratia spinosa (Aubl.) DC. & $\mathrm{S}$ & 19,2 & 6,7 & 1,0 & - \\
\hline Cecropiaceae & Cecropia glaziovii Snethl. & $\mathrm{S}$ & 5,0 & - & - & - \\
\hline Ebenaceae & Diospyros inconstans Jacq. & $\mathrm{S}$ & - & - & 1,0 & - \\
\hline Erythroxylaceae & Erythroxylum deciduum A. St.-Hil. & $\mathrm{M}$ & - & - & 1,0 & - \\
\hline \multirow{5}{*}{ Euphorbiaceae } & Alchornea triplinervia (Spreng.)Müll. Arg. & M & 14,1 & 3,3 & - & - \\
\hline & Gymnanthes concolor Spreng.. & $\mathrm{S}$ & 103,8 & 191,6 & 147,9 & 47,1 \\
\hline & Sebastiania brasiliensis Spreng. & $\mathrm{S}$ & - & - & 12,7 & 10,9 \\
\hline & $\begin{array}{l}\text { Sebastiania commersoniana (Baill.) L.B. Sm. \& } \\
\text { Downs }\end{array}$ & S & - & - & 6,9 & - \\
\hline & Tetrorchidium rubrivenium Poepp. \& Endl. & M & 5,1 & 2,2 & 1,0 & - \\
\hline \multirow{5}{*}{ Flacourtiaceae } & Banara tomentosa Clos & $\mathrm{S}$ & 5,1 & 2,2 & - & 7,5 \\
\hline & Casearia decandra Jacq. & S & - & - & 2,0 & 6,8 \\
\hline & Casearia silvestris $\mathrm{Sw}$. & S & - & 3,3 & 5,9 & 6,8 \\
\hline & Xylosma pseudosalzamannii Sleumer & S & - & - & 2,0 & - \\
\hline & Xylosma sp. & $\mathrm{S}$ & 5,1 & - & - & - \\
\hline Icacinaceae & Citronella paniculata (Mart.) R.A.Howard & $\mathrm{S}$ & - & 2,2 & 5,9 & 3,4 \\
\hline \multirow{5}{*}{ Lauraceaee } & Nectandra lanceolata Nees & $\mathrm{M}$ & - & - & 4,9 & 3,4 \\
\hline & Nectandra megapotamica (Spreng.) Mez & M & 19,2 & 32,3 & 34,3 & 25,2 \\
\hline & Ocotea diospyrifolia (Meisn.) Mez & M & 14,1 & 18,9 & 3,9 & 3,4 \\
\hline & Ocotea puberula (Rich.) Nees & M & - & - & - & 14,3 \\
\hline & Indeterminadas & $\mathrm{M}$ & - & 1,1 & 1,0 & 6,8 \\
\hline \multirow{4}{*}{ Caesalpiniaceae } & Apuleia leiocarpa (Vogel) J.F. Macbr. & $\mathrm{M}$ & 9,1 & 21,2 & 36,3 & 25,2 \\
\hline & Bauhinia forficata Link & $\mathrm{S}$ & 9,1 & 3,3 & 2,0 & - \\
\hline & Holocalyx balansae Micheli & M & 19,2 & 15,6 & 4,9 & 14,3 \\
\hline & Peltophorum dubium (Spreng.) Taub. & $\mathrm{M}$ & - & 4,5 & 1,0 & - \\
\hline
\end{tabular}


TABELA 2: Continuação ...

TABLE 2: Continued ...

\begin{tabular}{|c|c|c|c|c|c|c|}
\hline \multirow[t]{2}{*}{ Família } & & \multirow{2}{*}{$\begin{array}{c}\begin{array}{c}\text { Classe de } \\
\text { uso }\end{array} \\
\text { S }\end{array}$} & \multicolumn{2}{|c|}{$\mathrm{CCO}$} & \multicolumn{2}{|c|}{ SVA } \\
\hline & Calliandra foliolosa Benth. & & - & 2,2 & 6,9 & 3,4 \\
\hline \multirow{3}{*}{ Mimosaceae } & Inga marginata Willd. & $\mathrm{S}$ & 47,4 & 52,4 & 47 & 21,9 \\
\hline & Albizia sp. & M & 5,1 & - & 2,0 & 3,4 \\
\hline & Parapiptadenia rigida (Benth.) Brenan & M & - & 1,1 & 2,9 & 6,8 \\
\hline \multirow{3}{*}{ Papilionaceae } & Erythrina falcata Benth. & M & - & 2,2 & 1,0 & 3,4 \\
\hline & Myrocarpus frondosus Allemão & M & 5,1 & 6,7 & 11,8 & 14,3 \\
\hline & $\begin{array}{l}\text { Indeterminadas (Machaerium sp. Dalbergia sp. e } \\
\text { Lonchocarpus sp. Spreng.) }\end{array}$ & M & 19,2 & 24,5 & 52,9 & 36,2 \\
\hline Loganiaceae & Strychnos brasiliensis (Spreng.) Mart. & $\mathrm{S}$ & - & 1,1 & 32,3 & 14,3 \\
\hline Melastomataceae & Miconia cf. pusilliflora (DC.) Naudin & $\mathrm{S}$ & 5,1 & 2,2 & 1,0 & - \\
\hline \multirow{6}{*}{ Meliaceae } & Cabralea canjerana (Vell.) Mart. & $\mathrm{M}$ & 24,2 & 13,4 & 13,7 & 14,3 \\
\hline & Cedrela fissilis Vell & M & 5,0 & 11,1 & 7,8 & 10,9 \\
\hline & Guarea macrophylla Vahl & $\mathrm{S}$ & 5,0 & 10,0 & 2,0 & 3,4 \\
\hline & Trichilia catigua A. Juss. & $\mathrm{S}$ & 42,3 & 47,9 & 28,4 & 18,4 \\
\hline & Trichilia claussenii C. DC. & $\mathrm{S}$ & 117,9 & 103,6 & 142,0 & 51,2 \\
\hline & Trichilia elegans A. Juss. & $\mathrm{S}$ & - & 11,1 & 12,7 & 18,4 \\
\hline Monimiaceae & Hennecartia omphalandra J. Poiss. & $\mathrm{S}$ & - & 1,1 & 2,0 & - \\
\hline \multirow{3}{*}{ Moraceae } & Ficus guaranitica Chod. & $\mathrm{S}$ & - & 4,5 & 2,0 & - \\
\hline & Maclura tinctoria (L.) Don ex Steud. & M & - & 2,2 & - & - \\
\hline & $\begin{array}{l}\text { Sorocea bonplandii (Baill.) W.C. Burger, Lanj. } \\
\& \text { Boer }\end{array}$ & $\mathrm{S}$ & 146,1 & 201,7 & 56,8 & 43,7 \\
\hline \multirow{2}{*}{ Myrsinaceae } & Myrsine loefgrenii (Mez.) Imkhan. & $\mathrm{S}$ & - & 1,1 & - & - \\
\hline & Myrsine umbellata Mart. & $\mathrm{S}$ & - & - & 1,0 & 6,8 \\
\hline \multirow{9}{*}{ Myrtaceae } & Calyptranthes tricona $\mathrm{D}$. Legrand & S & 142,1 & 122,6 & - & - \\
\hline & Campomanesia guazumifolia (Cambess.) O. Berg & S & - & - & 2,9 & - \\
\hline & Campomanesia xanthocarpa O. Berg & S & 19,1 & 10,0 & 2,9 & - \\
\hline & Eugenia burkartiana (D. Legrrand) D. Legrand & $\mathrm{S}$ & - & 3,3 & 1,0 & - \\
\hline & Eugenia pyriformis Cambess. & S & 5,0 & 2,2 & 2,0 & - \\
\hline & Eugenia ramboi D. Legrand & $\mathrm{S}$ & 5,0 & 2,2 & - & - \\
\hline & Eugenia rostrifolia D. Legrand & M & 5,0 & 7,8 & - & - \\
\hline & Myrcianthes pungens (O. Berg) D. Legrand & M & - & 1,1 & 1,0 & - \\
\hline & Myrtaceae sp. & $\mathrm{S}$ & - & - & 1,0 & - \\
\hline Nyctaginaceae & Pisonia ambigua Heimerl & $\mathrm{S}$ & 9,1 & 6,7 & 8,8 & - \\
\hline \multirow{2}{*}{ Phytolaccaceae } & Phytolacca dioica $\mathrm{L}$. & $\mathrm{S}$ & - & 2,2 & 2,0 & - \\
\hline & Seguieria aculeata Jacq. & $\mathrm{S}$ & 24,2 & 15,6 & 2,0 & 3,4 \\
\hline Piperaceae & Piper gaudichaudianum Kunth. & $\mathrm{S}$ & - & 2,2 & - & - \\
\hline Polygonaceae & Ruprechtia laxiflora Meisn. & $\mathrm{M}$ & - & 1,1 & - & 3,4 \\
\hline Rosaceae & Prunus sellowii Koehne & $\mathrm{M}$ & - & 3,3 & 9,8 & 32,7 \\
\hline \multirow[t]{2}{*}{ Rubiaceae } & Randia armata (Sw.) DC. & $\mathrm{S}$ & - & - & 2,0 & - \\
\hline & Balfourodendron riedelianum (Engl.) Engl. & $\mathrm{M}$ & 36,3 & 4,5 & 19,6 & 14,3 \\
\hline \multirow[t]{3}{*}{ Rutaceae } & Helietta apiculata Benth. & M & - & - & 5,9 & 6,8 \\
\hline & Pilocarpus pennatifolius Lem. & $\mathrm{S}$ & 19,1 & 24,5 & 14,7 & 3,4 \\
\hline & Zanthoxylum rhoifolium Lam. & $\mathrm{M}$ & - & - & 2,9 & 6,8 \\
\hline
\end{tabular}


TABELA 2: Continuação ...

TABLE 2: Continued ...

\begin{tabular}{|c|c|c|c|c|c|c|}
\hline Família & Nome científico & $\begin{array}{l}\text { Classe de } \\
\text { uso }\end{array}$ & \multicolumn{2}{|c|}{$\mathrm{CCO}$} & \multicolumn{2}{|c|}{ SVA } \\
\hline \multirow{4}{*}{ Sapindaceae } & $\begin{array}{l}\text { Allophylus edulis (A. St.-Hil., Cambess. \& A. } \\
\text { Juss.) Radlk. }\end{array}$ & $\mathrm{S}$ & 5,0 & 1,1 & 6,9 & 10,9 \\
\hline & Cupania vernalis Cambess. & M & 5,0 & 1,1 & 3,9 & 3,4 \\
\hline & Diatenopteryx sorbifolia Radlk. & M & 5,0 & 3,3 & 4,9 & - \\
\hline & Matayba elaeagnoides Radlk. & $\mathrm{M}$ & - & - & - & 3,4 \\
\hline \multirow[t]{2}{*}{ Sapotaceae } & $\begin{array}{l}\text { Chrysophyllum gonocarpum (Mart. \& Eichl.) } \\
\text { Engl. }\end{array}$ & M & 5,0 & 14,5 & 36,2 & 36,1 \\
\hline & $\begin{array}{l}\text { Chrysophyllum marginatum (Hook \& Arn.) } \\
\text { Radlk. }\end{array}$ & M & 19,1 & 14,5 & 44,1 & 32,7 \\
\hline Simarourbaceae & Picrasma crenata (Vell.) Engl. & $\mathrm{S}$ & 9,1 & 2,2 & 5,9 & - \\
\hline Solanaceae & Solanum sanctaecatharinae Dunal & $\mathrm{S}$ & 5,0 & 2,2 & 4,9 & - \\
\hline Styracaceae & Styrax leprosus Hook. \& Arn. & $\mathrm{M}$ & - & 2,2 & 4,9 & 6,8 \\
\hline Tiliaceae & Luehea divaricata Mart. \& Zucc. & $\mathrm{M}$ & 9,1 & 5,6 & 3,9 & 14,3 \\
\hline Ulmaceae & Trema micrantha L. Blume & $\mathrm{S}$ & - & 1,1 & - & - \\
\hline \multirow{2}{*}{ Urticaceae } & Boehmeria caudata Sw. & $\mathrm{S}$ & - & - & 1,0 & - \\
\hline & Urera baccifera L. Gaudich. & $\mathrm{S}$ & 19,1 & 21,2 & 34,3 & 6,8 \\
\hline \multirow{2}{*}{ Verbenaceae } & Aloysia virgata (Ruiz \& Pav.) Juss. & $\mathrm{S}$ & 5,0 & 1,1 & - & - \\
\hline & Vitex megapotamica (Spreng.) Moldenke & $\mathrm{M}$ & 5,0 & 1,1 & 1,0 & 3,4 \\
\hline Total plantas ha ${ }^{-1}$ & & 1012 & & 1116 & 677 & \\
\hline
\end{tabular}

Considerando-se as vinte espécies com maior densidade florestal em cada fragmento, 17 delas foram igualmente detectadas pelos dois métodos amostrais dentro de cada fragmento, entre estas, Calypthranthes tricona exclusiva ao fragmento CCO. Da mesma maneira, se totalizada a densidade de plantas na relação das vinte espécies de maior densidade florestal classificadas pelo MQ em cada fragmento, obtiveram-se valores muito próximos entre os dois métodos amostrais, sendo 85,6 e $82,9 \%$ em CCO e 75,0 e 76,5\% em SVA respectivamente em resposta aos MQ e MP. Observa-se que ambos os métodos empregados foram eficazes na caracterização das espécies florestais abundantes.

Da mesma forma, as dez espécies do sub-bosque que apresentaram maior área basal em CCO representaram $87,7 \%$ (MP) e $87,6 \%$ (MQ) do total da área basal das espécies do sub-bosque florestal. Já, em SVA, as mesmas representaram $81,9 \%$ (MP) e 77,7\% (MQ). Nessa comparação, nove espécies em CCO e oito em SVA foram detectadas conjuntamente pelos dois métodos (Figura 2a). Por outro lado, considerando as dez espécies madeireiras com maior área basal, estas em CCO representaram 79,8 e 85,2\% em SVA, 73,6 e 73,2\% respectivamente nos MP e MQ, sendo que dessas espécies em ambos os remanescentes florestais sete foram detectadas conjuntamente pelos dois métodos (Figura 2b). 

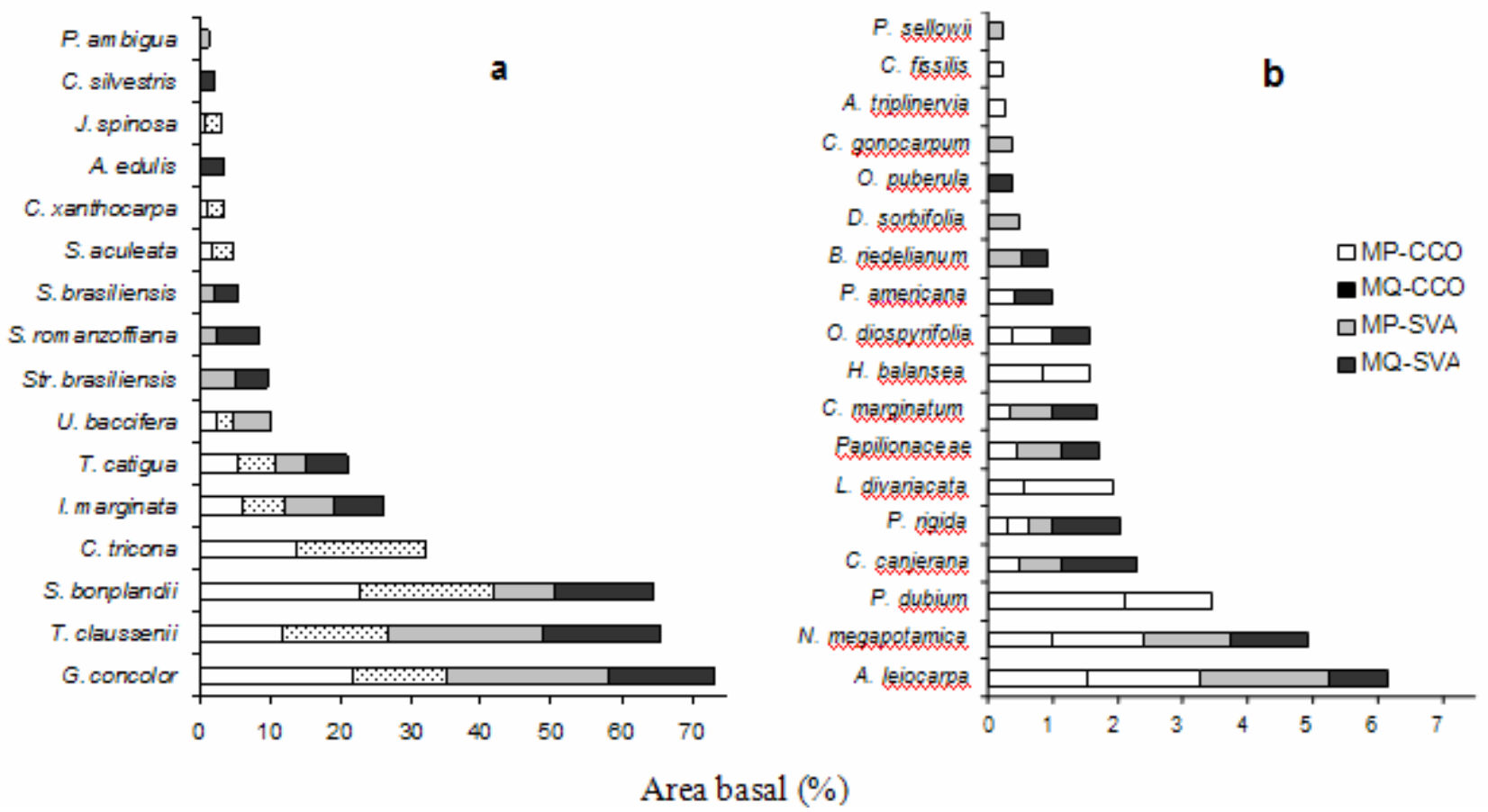

FIGURA 2: Relação das dez espécies de maior dominância relativa (área basal) ordenadas para os métodos de parcelas (MP) e pontos quadrantes (MQ), incluindo plantas com DAP $\geq 5 \mathrm{~cm}$ para as espécies do sub-bosque (a) e espécies de uso madeireiro (b). Detectados nos fragmentos São Valentin (SVA) e Capela Colégio (CCO) da Floresta Estacional Decidual do Alto-Uruguai, SC.

FIGURE 2: Ranking of the 10 species of highest relative dominance (basal area) classified for the methods of quadrate plots (MP) and point-centered quarter methods (MQ), includes plants with $\mathrm{DBH} \geq 5 \mathrm{~cm}$ for all subcanopy species (a) and for timber species (b). Detecting in the São Valentin (SVA) and Capela Colégio (CCO) fragments of the Seasonal Deciduous Forest in Alto-Uruguai, SC.

Várias espécies de uso madeireiro foram encontradas em alta densidade, sendo a maioria destas comuns e uniformemente distribuídas nos dois fragmentos. Entre tais espécies, as dominantes que caracterizaram a tipologia florestal foram: Nectandra megapotamica, Apuleia leiocarpa, Chrysophyllum marginatum, Ocotea diospyrifolia, Peltophorum dubium, Machaerium sp., Luehea divaricata, Holocalyx balansae, Cabralea canjerana, Pataganula americana, Balfourodendron riedelianum, Myrocarpus frondosus, Parapiptadenia rigida, Cordia trichotoma e Cedrela fissilis entre outras que, somadas nos dois remanescentes, representaram pelo menos 39 espécies identificadas. Da mesma forma, Ruschel et al. (2005) identificaram grande uniformidade quanto à composição das espécies madeireiras entre 13 fragmentos florestais do Alto-Uruguai e também reportaram a ocorrência de 63 espécies de uso madeireiro (RUSCHEL et al., 2003).

Comparações detalhadas entre os dois métodos de inventário e entre os dois remanescentes florestais também foram analisadas por meio dos índices de similaridade e diversidade. Nessas comparações detectouse que os índices de diversidade Shannon ( $\left.\mathrm{H}^{\prime}\right)$ e a equabilidade comunitária Pielou (J'), em ambos os fragmentos foram ligeiramente inferiores para o MP, quando comparados aos obtidos pelo MQ, com exceção o índice de diversidade de Simpson's que foi similar e ou levemente superior ao MP (Tabela 3). Os valores de diversidade de Shannon variaram de 3,03 a 3,58. Tais valores foram similares aos obtidos em outros estudos da mesma tipologia florestal (DIAS et al., 1992; RUSCHEL et al., 2006). Tomando-se exclusivamente as comparações entre remanescentes têm-se índices de diversidade superiores para o remanescente SVA, independentemente do método utilizado. Porém, especificamente para o grupo das espécies madeireiras, os índices de diversidade dos dois remanescentes são muito próximos entre si. O maior número de espécies detectados no fragmento florestal SVA em parte pode ser explicado pelo fato deste se encontrar em área de transição da Floresta Estacional Decidual - FED para a Floresta Ombrófila MistaFOM. Essa área de transição se caracteriza pelas altitudes próximas de $600 \mathrm{~m}$ e, nos limites desse fragmento, foi constatada a ocorrência natural da araucária (Araucaria angustifalia), espécie dominante da FOM 
(REITZ et al., 1978). A transição de tipologia florestal proporciona a mistura de espécies típicas às duas tipologias, conferindo assim uma maior diversidade.

Os índices quantitativos de similaridade como o de Morisita, Bray-Curtis e Perecentagem simples alcançaram valores altos para qualquer um dos métodos de inventário, variando de 76 a $100 \%$, em todas as categorias analisadas. No entanto, o índice de Jaccard revelou valores menores do que os índices estimados pelas demais metodologias empregadas. Se tomado o índice de Jaccard, observa-se a grande diferença entre os métodos de amostragem, já que tal índice considera apenas qualitativamente a floresta. Assim, os valores são diretamente influenciados pelo número de espécies (diversidade) e não pela riqueza, uma vez que as espécies de baixa densidade recebem igual peso que as espécies com alta densidade. Ao contrário, os métodos (Bray-Curtis, Percentagem Simples e Morisita) que consideram além da diversidade, a densidade de espécies (riqueza), detectaram alta similaridade entre os métodos amostrais MP e MQ. A importância da análise dos resultados produzidos por diferentes índices de similaridades indicou que aqueles índices que levam em conta a riqueza de espécies podem ser estimados por qualquer um dos dois métodos de amostragens. Adicionalmente, os diferentes valores revelados pelos diferentes métodos de inventário indicam que a amostragem para a avaliação da diversidade não foi completamente suficiente.

TABELA 3: Índices de diversidade de espécies de Shannon (H'), recíproca Simpson's, equabilidade da diversidade comunitária J' Pielou e índices de similaridade de Jaccard, Morisita, Bray-Curtis e Pecentagem Simples, estimados pelos métodos de amostragens de parcelas (MP) e pontos quadrantes (MQ), para plantas com DAP $\geq 5 \mathrm{~cm}$ e nas categorias sub-bosque e madeiráveis, avaliados nos fragmentos São Valentin (SVA) e Capela Colégio (CCO) da Floresta Estacional Decidual do Alto-Uruguai, SC.

TABLE 3: Indexes of species diversity, Shannon $\left(\mathrm{H}^{\prime}\right)$, reverse of Simpson's, evenness of Pielou ( $\left.\mathrm{J}^{\prime}\right)$, and similarity indices, Jaccard, Morisita, Bray-Curtis and Simple Percentage, estimated with the use of the quadrate plots (MP) and point-centered quarter methods (MQ), for plants with $\mathrm{DBH} \geq 5 \mathrm{~cm}$ grouped as subcanopy and timber species evaluated in the São Valentin (SVA) and Capela Colégio (CCO) fragments of the Seasonal Deciduous Forest in Alto-Uruguai, SC.

\begin{tabular}{|c|c|c|c|c|c|c|}
\hline \multirow{2}{*}{ Índices } & \multicolumn{2}{|c|}{ Todas } & \multicolumn{2}{|c|}{ Sub-bosque } & \multicolumn{2}{|c|}{ Madeiráveis } \\
\hline & MP & MQ & MP & MQ & MP & MQ \\
\hline H' Shannon - CCO & 3,03 & 3,07 & 2,39 & 2,46 & 2,98 & 3,07 \\
\hline Simpson's - CCO & 1,10 & 1,08 & 1,63 & 1,14 & 1,07 & 1,08 \\
\hline J' Pielou - CCO & 0,71 & 0,81 & 0,65 & 0,77 & 0,86 & 0,88 \\
\hline Jaccard - CCO & \multicolumn{2}{|c|}{0,55} & \multicolumn{2}{|c|}{0,54} & \multicolumn{2}{|c|}{0,58} \\
\hline Morisita - CCO & \multicolumn{2}{|c|}{0,99} & \multicolumn{2}{|c|}{0,99} & \multicolumn{2}{|c|}{1,0} \\
\hline Bray-Curtis - CCO & \multicolumn{2}{|c|}{0,89} & \multicolumn{2}{|c|}{0,89} & \multicolumn{2}{|c|}{0,86} \\
\hline Percent. Simples - CCO & \multicolumn{2}{|c|}{0,89} & \multicolumn{2}{|c|}{0,90} & \multicolumn{2}{|c|}{0,88} \\
\hline H' Shannon - SVA & 3,34 & 3,58 & 3,63 & 2,68 & 2,84 & 3,03 \\
\hline Simpson's - SVA & 1,07 & 1,04 & 1,14 & 1,10 & 1,09 & 1,06 \\
\hline J'Pielou - SVA & 0,77 & 0,90 & 0,70 & 0,87 & 0,80 & 0,90 \\
\hline Jaccard - SVA & \multicolumn{2}{|c|}{0,60} & \multicolumn{2}{|c|}{0,49} & \multicolumn{2}{|c|}{0,68} \\
\hline Morisita - SVA & \multicolumn{2}{|c|}{0,89} & \multicolumn{2}{|c|}{0,90} & \multicolumn{2}{|c|}{1,0} \\
\hline Bray-Curtis - SVA & \multicolumn{2}{|c|}{0,79} & \multicolumn{2}{|c|}{0,76} & \multicolumn{2}{|c|}{0,85} \\
\hline Percent. Simples - SVA & \multicolumn{2}{|c|}{0,81} & \multicolumn{2}{|c|}{0,79} & \multicolumn{2}{|c|}{0,85} \\
\hline
\end{tabular}

\section{CONCLUSÕES}

No presente trabalho, identificaram-se 91 espécies, das quais aproximadamente a metade são espécies do sub-bosque florestal (sem uso madeireiro). Destas, um pequeno grupo formado de menos de cinco espécies, foram dominantes nos dois remanescentes, destacando-se Sorocea bonplandii, Gimnanthes concolor e Trichilia claussenii. Por sua vez, na estrutura do dossel florestal, foram identificadas pelo menos 39 espécies de uso madeireiro, sendo as mais abundantes Apuleia leiocarpa, Nectandra megapotamica, Ocotea diospyrifolia, Holocalyx balansae, Peltophorum dubium e Cabralea canjerana. As espécies de maior densidade e área basal foram similares em ambos os fragmentos florestais, independentes do método usado. 
Ressalta-se que a riqueza de espécies madeireiras presentes na FED, contrasta com o pequeno número de fragmentos que restaram neste ecossistema e estes fragmentos são as últimas fontes de sementes para a recolonização de áreas abandonadas ou degradadas, como também para uso em programas de melhoramento genético de essências florestais nativas e banco de sementes in situ. No entanto, sugere-se a conservação de todos esses fragmentos, considerando-os importantes e necessários para assegurar a conservação da ameaçada biodiversidade contida nestes. Além da sua conservação, sugere-se a ampliação e criação de conexões entre esses remanescentes, para assim garantir a manutenção da diversidade ainda existente.

Os vários parâmetros e índices usados para fins comparativos tanto entre métodos de amostragem como entre remanescentes florestais apontam que o aumento do número amostral é a melhor estratégia para inventários amostrais. Na comparação entre métodos de amostragem, com o Método de Parcelas é possível identificar uma maior riqueza florística, embora ambos os métodos demonstrem resultados similares para caracterização da vegetação dominante. Contudo, o uso simultâneo dos dois métodos permitiu inventariar uma maior diversidade de espécies, contribuindo assim para a maior cobertura amostral dos remanescentes amostrados.

\section{AGRADECIMENTOS}

Ao Conselho Nacional de Desenvolvimento Científico e Tecnológico (CNPq/Brasil), pelas bolsas concedidas aos autores. Aos Professores Ademir Reis (CCB-USFC) e Marcos Sobral (UFRGS), pela identificação do material botânico. Pelo apoio de campo, Antônio R. Ruschel, Erasmo Tiepo, Elefor A. G. Fagundes.

\section{REFERÊNCIAS BIBLIOGRÁFICAS}

BRAUN-BLANQUET, J. Fitossociologia: bases para el estudio de las comunidades vegetales. Madrid: H. Blume, 1979. $820 \mathrm{p}$.

CONSEMA - Conselho Estadual do Meio Ambiente. Decreto Estadual No 42.099/2002. Espécies da flora ameaçadas de extinção do Rio Grande do Sul, 2002. citation on computers documents. Disponível em: (http://www.sema.rs.gov.br/sema/html/espec.htm) > Acesso em: 15 de junho de 2006.

COTTAM, G.; CURTIS, J. T. The use of distance measures in phythological sampling. Ecology, Durham, v.37, p. 451460, 1956.

CRONQUIST, A. The evolution and classification of flowering plants. New York: The New York Botanical Garden, 1988. $555 \mathrm{p}$.

DIAS, L. L. et al. Levantamento florístico de uma área de mata subtropical no Parque Estadual do Turvo, Tenente Portela, RS. In: CONGRESSO NACIONAL SOBRE ESSÊNCIAS NATIVAS, 2., 1992, São Paulo. Anais.... Instituto Florestal: São Paulo, 1992. v. 4, p. 339-346.

GIBBS, P. E.; LEITÃO FILHO, H. F.; JABBOTT, R. J. Application of the point-centred quarter method in a florístic survey of an area of gallery forest at Mogi-Guaçu, SP, Brazil. Revista Brasileira de Botanica, São Paulo, v. 3, n. 1, p. 17-22, jan./jun. 1980.

KLEIN, R. M. Árvores Nativas da Floresta Subtropical do Alto Uruguai. Sellowia, v. 24, p. 09-62, 1972.

KREBS, C. J. Ecological Methodology. New York: Harper e Row, 1989. 654 p.

IBGE - Instituto Brasileiro de Geografia e Estatística. Vegetação e geografia do Brasil: Região Sul. Rio de Janeiro: 1990. 300 p. v. 2.

LORENZI, H. Árvores brasileiras: manual de cultivo de plantas arbóreas nativas do Brasil. Nova Odessa: Plantarum, 1998. v, 1-2.

MAGURRAN, A. E. Ecological diversity and its measurement. New Jersey: Princeton University Press, 1988.179 p. MARTINS, F. R. Estrutura de uma Floresta Mesófila. Campinas: UNICAMP, 1993. 243 p.

METZGER, J. P; GOLDENBERG, R.; BERNACCI, L. C. Diversidade e estrutura de fragmentos de mata mesófila semidecídua submontana do rio Jacaré-Pepira (SP). Revista Brasileira de Botânica, v. 21, n. 3, p. 321-330, 1998.

MÜLLER-DOMBOIS, D.; ELLEMBERG, H. Aims and methods of vegetation ecology. New York: John Willey e Sons, 1974. 547 p.

PIAZZA, W. F. A colonização de Santa Catarina. 3. ed. Florianópolis: Lunardelli, 1994. 372 p.

POLLARD, J. H. On distance estimators of density in randomly distributed forests. Biometrics, v. 27, p. 991-1002, 1971. 
RAMBO, B. Der Regenwald am oberen Uruguay. Sellowia, v. 7, p. 183-223, 1956.

REIS, A. Manejo e conservação das florestas catarinenses. 1993. 137 f. Tese (Concurso de de Professor Títular de Botânica Aplicada) - Universidade Federal de Santa Catarina, Florianópolis.

REITZ, R.; KLEIN, R. M.; REIS, A., Projeto Madeira de Santa Catarina. Sellowia, Itajai, v 28/30, p. 1-320, 1978.

RUSCHEL, A. R. et al. Valuation and characterization of the timber species in remnants of the Alto Uruguai River ecosystem, Southern Brazil. Forest Ecology and Management, Amsterdam, v. 217, p. 103-116, 2005.

RUSCHEL, A. R. et al. Evolução do Uso e Valorização das Espécies Madeiráveis da Floresta Estacional Decidual do Alto-Uruguai-SC. Ciência Florestal, Santa Maria, v. 13, n. 1, p. 153-166, jul./dez. 2003.

RUSCHEL, A. R.; NODARI, R. O.; MOERSCHBACHER, B. M. Woody plant species richness in the Turvo State park, a large remnant of deciduous Atlantic forest, Brazil. Biodiversity and Conservation, Amsterdam, DOI: 10.1007/s10531-006-9044-7, 2006.

SILVA, L. O. et al. Levantamento Florístico e Fitossociológico em duas áreas de cerrado senso stricto no Parque Estadual de Serra de Caldas Novas, Goiás. Acta Botânica Brasílica, Porto Alegre, v. 16, n. 1, p. 43-53, jan../abr. 2002. SPARKS, J. C.; MASTERS, R. E.; PAYTON, M. E. Comparative Evaluation of Accuracy and Efficiency of Six Forest Sampling Methods. Proccedings Oklahoma Academy Science, v. 82, p. 49-56, 2002.

UBERTI, A. A. A. Santa Catarina: Proposta de Divisão Territorial em Regiões Edafoambientais Homogêneas. 2005. 185 f.. Tese (Doutorado em Engenharia Civil) - Universidade Federal de Santa Catarina, Florianópolis.

WAllauer, J. P.; ALBUQUERQUE, E. P. Lista preliminar dos mamíferos observados no Parque Florestal do Turvo, Tenente Portela, Rio Grande do Sul, Brasil. Roessiléria, v. 8, n. 2, p. 179-185, 1986. 